\title{
Correspondence
}

To the Editors,

\section{Magnesium sulphate in the management of birth asphyxia}

Sri Lanka Journal of Child Health, 2017; 46(3): 297-299

DOI: http://dx.doi.org/10.4038/sljch.v46i3.8341

(Key words: Magnesium sulphate, management, birth asphyxia)

We read with much interest the article by Sreenivasa et $a l^{1}$ in the June 2017 issue of your journal. We would like to make a few comments, clarification of which is expected to benefit the general readers of the journal.

1. The authors have taken a sample size of 100 term neonates (50 study group and 50 control group) but the basis of this sample size calculation is not provided. This is of importance as the power of the study is dependent on the sample size ${ }^{2}$.

2. The basis for allocation of neonates in the study or control group is not mentioned. So, whether this allocation was based on any sort of randomization or was solely on the basis of researcher's preference which introduces selection bias. Furthermore, the study being 'open label', the measures taken to reduce observer bias is also not mentioned ${ }^{3}$.

3. The methodology states that "Routine protocol for asphyxiated babies in the neonatal intensive care unit (NICU) was followed in all 100 neonates". But this 'routine protocol' needed to be described in more detail as the practices across the different NICUs likely to differ pertaining to the management of neonates with perinatal asphyxia, such as use of use of therapeutic hypothermia, etc. This is important in comparing results of similar studies across the regions. Moreover, it is imperative to know the protocol followed for feeding of these newborns, i.e. the criteria to start them on nasogastric tube feeding, spoon feeding or direct breast feeding and use of antiepileptics for seizure, as they were found to be significantly different in the study and control groups.

4. Table 2 describes that $25.5 \%$ neonates in the study group and $47.8 \%$ in the control group had abnormal neurological findings at discharge. But it is also presented in the same table presents that 'the mean duration of recovery from neurological abnormality' to be 3.56 and 5.01 days in the study and control groups respectively. This raises the question whether neonates in the study group were discharged at a mean duration less than 3.56 days and then followed up to study their 'recovery' from neurological abnormalities. This is very unlikely as the time to reach spoon feeding in this group was 3.9 days! Same would also apply for the control group.

5. The title and objective of the article indicate the intentions to assess the "efficacy of intravenous (IV) magnesium sulphate in treating birth asphyxia and improving short term neurological outcome". But the authors did not provide the duration of this "short term" for which these neonates were followed, actually even the duration of hospital stay in the two groups is not mentioned.

6. The Table 2 presents a total of 7 mortalities (3 in the study group and 4 in the control group) out of total 100 participants. This makes a total mortality rate of $7 \%$ but in the discussion it is mentioned that "The overall mortality in the present study was $14 \%$ "!

7. There is mention that history of antenatal risk factors for perinatal asphyxia and detailed anthropometry of the neonates were recorded but no such data is presented.

\section{References}

1. Sreenivasa B, Lokeshwari K, Joseph N. Role of magnesium sulphate in management and prevention of short term complications of birth asphyxia. Sri Lanka Journal of Child Health, 2017; 46(2): 148-51.

2. Jones SR, Carley S, Harrison M. An introduction to power and sample size estimation. Emergency Medicine Journal 2003; 20(5): 453-8.

3. Pandis N. Sources of bias in clinical trials. American Journal of Orthodontics and Dentofacial Orthopedics 2011; 140(4): 595-6.

Anirban Mandal ${ }^{1}$, Puneet Kaur Sahi ${ }^{2}$

${ }^{1}$ Sitaram Bhartia Institute of Science and Research, India, ${ }^{2}$ Kalawati Saran Children's Hospital, India 


\section{$\underline{\text { Response by authors of article }}$}

To the Editors,

Response to comment No. 1

A minimum sample size of 100 cases was required using an incidence of birth asphyxia as 78/2001 of total admissions in 6 months at our institution at a significance level of 0.05 .

Response to comment No. 2

Patients were randomly allocated into two groups of 50 each.

Response to comment No. 3

- The baby was put under the radiant warmer after initial resuscitation of the baby. Maintained normal temperature of the baby. Immediate clinical assessment was made. All babies were started on intravenous fluids (10\% dextrose), about two-third of the maintenance fluid due to the possibility of syndrome of inappropriate $\mathrm{ADH}$ secretion.

- Baseline investigations like blood sugar, serum calcium, complete blood count, serum electrolytes and blood gases were done. If the capillary refill time was more than 2 seconds or if there was metabolic acidosis, volume expansion with normal saline (or Ringer's lactate) $10 \mathrm{ml} / \mathrm{kg}$ over 5-10 minutes was instituted. This was repeated, if required. Decrease in vascular tone results in relative hypovolemia (preload) in babies with asphyxia. Attention to perfusion is the single most important component of therapy of asphyxiated neonates at this stage. Subsequent management included continuous monitoring, maintaining oxygenation and ventilation, maintaining adequate perfusion, volume expansion and vasopressors.

- The target $\mathrm{PaO}_{2}$ is 80-100torr in term babies and 60-80torr in preterm infants. Pulse oximeter saturation values are maintained in the $90-95 \%$ range in term babies and $90-93 \%$ in the preterm infants. The target $\mathrm{PaCO}_{2}$ value is $40-45$ torr. The markers of normal perfusion are normal blood pressure, capillary refill time of less than 2 seconds, normal urine output, and absence of metabolic acidosis. The BP should be maintained in the upper normal range. Maintain normal blood glucose and calcium level.

- The anticonvulsant of choice for controlling seizures was phenobarbitone. The initial loading dose is $20 \mathrm{mg} / \mathrm{kg}$, intravenously slowly over 20 minutes. If there is no response, two additional doses of $10 \mathrm{mg} / \mathrm{kg}$ can be given every 15 minutes. If convulsions are still uncontrolled, phenytoin sodium should be added in a dose of $20 \mathrm{mg} / \mathrm{kg}$ intravenously slowly over 20 minutes. Maintenance therapy of both phenobarbitone and phenytoin are started 12 hours later in a dose of $5 \mathrm{mg} / \mathrm{kg} /$ day in a single dose. Generally, one or both these anticonvulsants are effective. For intractable seizures clonazepam, midazolam, paraldehyde, and valproate were tried. For management of seizures same protocol used for both the groups.

- In our NICU setup we did not used therapeutic hypothermia for management of birth asphyxia.

- Management of cases and controls were similar in all aspects except that we used magnesium sulphate in cases in addition to routine treatment.

- Criteria to start type of feeding: If an infant was well and making sucking movements, and was not in respiratory distress direct breast feeding is attempted, although most infants weighing $<1500 \mathrm{~g}$ require tube feeding because they are unable to coordinate breathing, sucking and swallowing. Intestinal tract readiness for feeding may be determined by active bowel sounds, passage of meconium and the absence of abdominal distension, bilious gastric aspirates and emesis.

Response to comment No. 4

Please don't confuse total duration of stay in hospital due to birth asphyxia and mean duration of recovery from neurological abnormality in patients who developed neurological abnormality as a consequence of birth asphyxia.

Response to comment No. 5

Patients were studied from the day of admission till the discharge from hospital. 


\begin{tabular}{|c|c|c|}
\hline & Duration of hospital stay \\
\hline & Number & Mean \pm SD \\
\hline Cases & 50 & $8.733 \pm 2.065$ \\
\hline Controls & 50 & $9.250 \pm 3.144$ \\
\hline$p=0.290$ & &
\end{tabular}

There was no statistically significant difference in the duration of hospital stay between cases and controls, hence the mean duration of hospital stay was similar.

Response to comment No. 6

Sorry. Its $7 \%$ only. Human error!!

\section{Response to comment No. 7}

- Among cases the mean age of mothers was 21.9 years in which $72 \%$ were primiparous and rest multiparous. Other risk factors associated with perinatal asphyxia in cases included meconium stained amniotic fluid (45\%), prolonged labour $(21 \%)$, premature rupture of membrane $(20 \%)$, cord around the neck $(10 \%)$ and pregnancy induced hypertension $(10 \%)$.

- Among controls the mean age of mothers was 21.5 years in which $76 \%$ were primiparous and rest multiparous. Other risk factors associated with perinatal asphyxia in controls included meconium stained amniotic fluid (48\%), premature rupture of membrane $(25 \%)$, cord around the neck (12\%), prolonged labour (18\%) and pregnancy induced hypertension (11 $\%)$.
- There was no statistically significant difference between cases and controls in terms of risk factors associated with perinatal asphyxia.

- Most of the subjects, that is, $93 \%$ of cases and $96 \%$ of controls were appropriate for gestational age. Only 5\% of cases and 3\% of controls were small for gestational age. Only one case was large for gestational age. There was no statistically significant difference between cases and controls in terms of weight for gestational age. Hence both study groups were similar in terms of assessment of weight for gestational age

*B Sreenivasa ${ }^{1}$, K Lokeshwari ${ }^{1}$, Nivil Joseph ${ }^{2}$

${ }^{1}$ Basaveshwara Medical College Hospital and Research Centre, Chitradurga, India, ${ }^{2} S J M$ College of Pharmacy, Chitradurga, India

*Correspondence: sreenivasab@ymail.com 